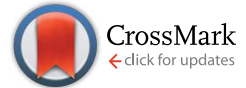

Cite this: RSC Adv., 2016, 6, 34977

\title{
One-pot, mouldable, thermoplastic resins from poly(propylene carbonate) and poly(caprolactone triol) $\uparrow$
}

\begin{abstract}
S. Spoljaric and J. Seppälä*
Co-polymers of poly(propylene carbonate) (PPC) and poly(caprolactone triol) (PCLT) were synthesised via a simple yet effective one-pot, two-step method, without the need for a catalyst or solvent. Successful synthesis was confirmed via ${ }^{1} \mathrm{H}$ NMR and ATR-FT-IR. The co-polymers displayed increased thermal stability. Covalent bonding between the functionalised PCLT and PPC enhanced moduli and strength, while also reducing creep and flow within PPC. Co-polymers displayed increased storage moduli, glass transition temperatures and damping properties. Additionally, PCLT-PPC co-polymers were flexible and easily moulded into shapes while maintaining their form and structural integrity. By imparting a relatively small concentration of functionalised-PCLT, the existing material properties (damping), as well as those previously seen as a hindrance to greater PPC application (poor thermal stability, cold flow, poor strength) were enhanced, while new characteristics (mouldability) were introduced.
\end{abstract}

Received 18th March 2016

Accepted 28th March 2016

DOI: 10.1039/c6ra07191k

www.rsc.org/advances

\section{Introduction}

Synthetic polymers, mostly prepared from non-renewable fossil fuels, have been a staple material in many applications and industries for well over a century. However, the rapid consumption of petroleum, coupled with factors such as pollution and growing environmental consciousness have shifted the research focus towards the development of biodegradable polymers from renewable feedstocks. ${ }^{1,2}$ One such polymer is poly(propylene carbonate) (PPC), a thermoplastic aliphatic polyester which is prepared via copolymerisation of carbon dioxide $\left(\mathrm{CO}_{2}\right)$ and propylene oxide. ${ }^{3}$ Apart from providing a value-added pathway of utilising $\mathrm{CO}_{2}$, PPC's other favourable attributes include biodegradability, ${ }^{4}$ renewability, cost effectiveness, ${ }^{5}$ transparency ${ }^{6}$ and exceptional barrier ${ }^{7}$ and elastic/ductile properties. Despite its mass appeal and the recent reporting of large-scale production capabilities, ${ }^{8}$ its greater applicability is limited by its poor mechanical strength and low glass transition temperature $T_{\mathrm{g}}$ of $\sim 40{ }^{\circ} \mathrm{C},{ }^{9}$ often leading to cold flow under ambient conditions. ${ }^{10}$ Thus, imparting strength, rigidity and structural integrity into PPC is a major consideration when further applicability and use is concerned.

The introduction of branching or crosslinking (facilitated via blending or co-polymerisation with compatible polymers) is a convenient and efficient approach to overcoming PPC's hindrances. In particular, the introduction of branched structures

Polymer Technology Research Group, Department of Biotechnology and Chemical Technology, School of Chemical Technology, Aalto University, P.O Box 16100, Aalto, Finland.E-mail: jukka.seppala@aalto.fi

$\dagger$ Electronic supplementary information (ESI) available. See DOI: 10.1039/c6ra07191k is preferred in order to retain the thermoplastic nature of PPC. One of the most promising candidates to fulfil this role is poly(caprolactone triol) (PCLT), having been successfully utilised previously to toughen poly(lactic acid) ${ }^{\mathbf{1 1} 12}$ and as a component in various polyurethane systems. ${ }^{\mathbf{1 3 , 1 4}}$ Furthermore, PCLT is biodegradable, ${ }^{15}$ biocompatible ${ }^{16}$ miscible with a wide range of polymers and displays superior UV resistance properties. Although poly(caprolactone) (PCL) is synthesised from petroleum-derived sources, ${ }^{17}$ potential exists to prepare PCL precursors from renewable sources, such as glucose and D-fructose. ${ }^{18,19}$ This drive towards utilising polymers from renewable resources has produced limited research regarding the preparation of PPC-PCL blends and copolymers ${ }^{20}$ exploring possible applications including nanofibrilar scaffolds ${ }^{21}$ and toughening agents for epoxy resins. ${ }^{22}$ However, the aforementioned compositions utilise linear poly( $\varepsilon$-caprolactone) rather than the triol form, providing opportunity to probe the reinforcing ability of PCLT. Likewise, the mechanical and rheological data regarding PPC-PCLT co-polymers is rather limited; this provides great potential in exploring possible alternative applications, especially with regard to films.

Herein, the synthesis and characterisation of PPC-PCLT copolymers is described. Co-polymer synthesis consisted of a onepot, two-step approach; firstly, a polycondensation reaction between PCLT and either succinic or sebacic acid introduced carboxylic acid moieties to the chain ends. The second step involved an esterification reaction between the carboxylated PCLT pre-polymer and hydroxyl end groups of PPC. The copolymer synthesis proved to be a simple yet effective method, requiring no solvent or catalyst. To the best of the authors' knowledge, this is the first reported synthesis of such co- 
a)<smiles>[R6]CC(CC)(C[OH+])C[OH2+]</smiles><smiles></smiles>

b)

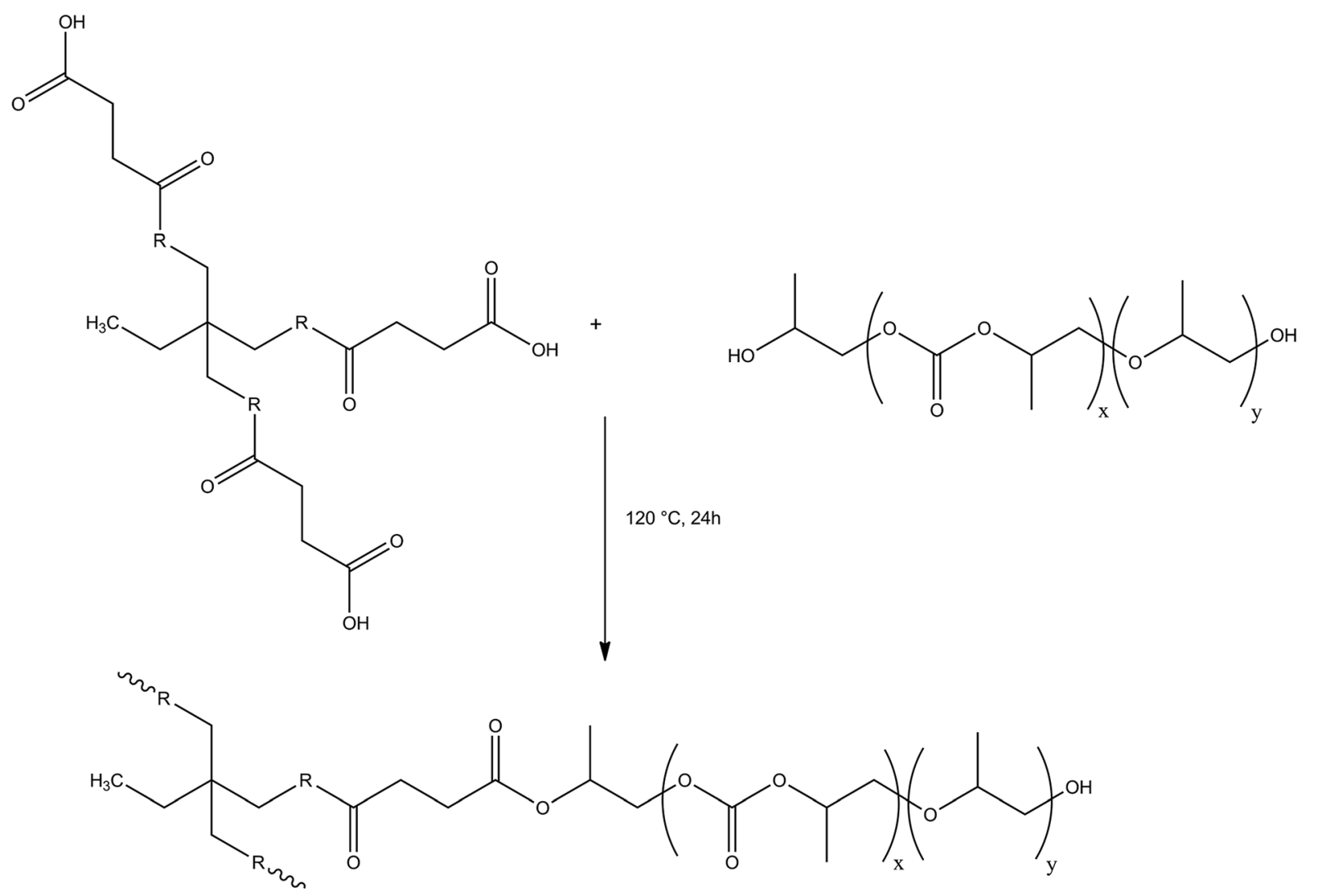

Scheme 1 (a) Synthesis of PCLT-dicarboxylic acid pre-polymer, (b) synthesis of PPC-PCLT co-polymer. 
polymers. The mechanical, rheological, thermal and morphological properties of the co-polymers were characterised.

\section{Experimental}

\section{Materials}

Poly(propylene carbonate) (PPC, $M_{\mathrm{w}} \sim 50000$, cyclic propylene carbonate content 4.0-5.5 mol\%), poly(caprolactone triol) (PCLT, $\left.M_{\mathrm{w}} \sim 900\right)$, succinic acid ( $\left.\geq 99.0 \%\right)$ and sebacic acid (99\%) were purchased from Sigma Aldrich, USA and used as received.

\section{Synthesis of PPC-PCLT co-polymers}

The synthesis of the PPC-PCLT co-polymers (using succinic acid as an example) is summarised in Scheme 1. PCLT and either succinic- or sebacic acid were charged into a twin-neck $100 \mathrm{~mL}$ round-bottom flask. The molar ratio between PCLT and succinic/ sebacic acid was either $1: 1$ or $1: 3$. The contents of the flask were stirred at $160^{\circ} \mathrm{C}$ for $1 \mathrm{~h}$, after which the pre-polymer mixture was cooled to $120^{\circ} \mathrm{C}$. PPC was added to the flask (PCLT : PPC molar ratio of $1: 3$ ) and the reaction mixture was stirred for $24 \mathrm{~h}$. Films for subsequent use and analysis were prepared using a heated press (TP400 Table Press, Fortune Holland, Netherlands). Films were heated at $100{ }^{\circ} \mathrm{C}$, held under $150 \mathrm{kPa}$ of pressure for $5 \mathrm{~min}$ then slowly cooled to ambient. The nomenclature and composition of the PPC-PCLT-copolymers is summarised in Table 1.

\section{Characterisation of PPC-PCLT co-polymers}

Size exclusion chromatography (SEC). SEC was performed utilising a Waters Associates system equipped with a Waters 717Plus Satellite autosampler, a Waters 510 HPLC solvent pump, four linear PL gel columns $\left(10^{4}, 10^{5}, 10^{3}\right.$, and $\left.100 \AA\right)$ connected in series, and a Waters 2414 differential refractometer. The numberaverage molecular weight $\left(M_{\mathrm{n}}\right)$, weight-average molecular weight $\left(M_{\mathrm{w}}\right)$ and molecular weight distribution of the PPC-PCLT copolymers were determined against polystyrene standards at $40{ }^{\circ} \mathrm{C}$. Tetrahydrofuran was used as the eluent and was delivered at a flow rate of $0.6 \mathrm{~mL} \mathrm{~min}{ }^{-1}$. Specimens were dissolved in THF at a concentration of $10 \mathrm{ppm}$. The injection volume was $200 \mu \mathrm{L}$.

${ }^{1} \mathbf{H}$ NMR. ${ }^{1} \mathrm{H}$ NMR spectra were recorded with a Bruker AVANCE-III $400 \mathrm{MHz}$ spectrometer operating at $400.13 \mathrm{MHz}$ for ${ }^{1} \mathrm{H}$. The spinning speed of samples was $8000 \mathrm{~Hz}$, contact time 2 $\mathrm{ms}$ and delay between pulses $5 \mathrm{~s}$. Samples were dissolved in chloroform- $\mathrm{d}_{1}$ (Fluka Chemical) with deuteration at no less than $99.8 \%$, in $5 \mathrm{~mm}$ NMR tubes, at room temperature.

Attenuated total reflectance infrared spectroscopy (ATR-FTIR). A Unicam Mattson 3000 FT-IR spectrometer equipped with

Table 1 PPC-PCLT co-polymer nomenclature and composition

\begin{tabular}{llll}
\hline $\begin{array}{l}\text { Sample } \\
\text { name }\end{array}$ & $\begin{array}{l}\text { Dicarboxylic } \\
\text { acid }\end{array}$ & $\begin{array}{l}\text { PCLT }: \text { dicarboxylic } \\
\text { acid ratio (mol) }\end{array}$ & $\begin{array}{l}\text { PCLT : PPC } \\
\text { ratio (mol) }\end{array}$ \\
\hline CP-Seb1 & Sebacic acid & $1: 1$ & $1: 3$ \\
CP-Seb3 & Sebacic acid & $1: 3$ & $1: 3$ \\
CP-Suc1 & Succinic acid & $1: 1$ & $1: 3$ \\
CP-Suc3 & Succinic acid & $1: 3$ & $1: 3$
\end{tabular}

PIKE Technologies GladiATR (with diamond crystal plate) was used to characterize the chemical structure of the PPC-PCLT copolymers. All spectra were scanned within the range 400 to 4000 $\mathrm{cm}^{-1}$, with a total of 16 scans and a resolution of $32 \mathrm{~cm}^{-1}$.

Thermogravimetric analysis (TGA). A TA Instruments Q500 Thermogravimetric Analyzer was used to characterize the thermal stability and degradation behavior of the filaments. Samples of $\sim 10 \mathrm{mg}$ were heated from 50 to $750{ }^{\circ} \mathrm{C}$ at $20^{\circ} \mathrm{C} \mathrm{min}{ }^{-1}$ in an inert environment provided by a $20 \mathrm{~mL} \mathrm{~min}^{-1}$ nitrogen purge.

Stress-strain analysis. Stress-strain analysis was performed using an Instron Universal Testing Instruments, Model 33R4204, with a $100 \mathrm{~N}$ static load attached. A strain rate of $10 \mathrm{~mm} \mathrm{~min}^{-1}$ was applied to the test specimens (average dimensions: $20.00 \times$ $5.30 \times 0.20 \mathrm{~mm}^{3}$ ) at $23{ }^{\circ} \mathrm{C}$ and $50 \%$ relative humidity. The results presented are the average of five parallel measurements.

Creep-recovery analysis. A TA Instruments Q800 Dynamic Mechanical Analyser operating in tensile mode was used to analyse the creep-recovery behaviour of the polymers. Films (average dimensions: $20.00 \times 5.30 \times 0.20 \mathrm{~mm}^{3}$ ) were subjected to an applied stress of $0.2 \mathrm{MPa}$ for $30 \mathrm{~min}$, followed by a recovery period of $120 \mathrm{~min}$ with $0.01 \mathrm{MPa}$ applied stress. The applied stress chosen was within the linear-viscoelastic region of all polymers. Tests were conducted at ambient temperature $\left(25^{\circ} \mathrm{C}\right)$ and all results presented are the average of triplicate measurements.

Dynamic mechanical analysis (DMA). DMA was performed using a TA Instruments Q800 Dynamic Mechanical Analyser operating in tensile mode. A frequency of $1 \mathrm{~Hz}$ and amplitude of $15 \mu \mathrm{m}$ were applied to the test specimens (average dimensions: $20.00 \times 5.30 \times 0.20 \mathrm{~mm}^{3}$ ). The storage modulus $\left(E^{\prime}\right)$ and loss tangent $(\tan \delta)$ were measured as a function of temperature from -100 to $120{ }^{\circ} \mathrm{C}$ at a heating rate of $3{ }^{\circ} \mathrm{C} \mathrm{min}^{-1}$.

\section{Results and discussion}

\section{Co-polymer structure and chemistry}

SEC. The SEC data of the pure polymers and co-polymers is summarised in Table 2. A reduction in both number average and weight average molar mass was observed for the co-polymers; similar behaviour has been previously reported ${ }^{23,24}$ and may be attributed to the occurrence of chain unzipping within PPC during synthesis at elevated temperatures (i.e. $120{ }^{\circ} \mathrm{C}$ ).

${ }^{1} \mathrm{H}$ NMR. Fig. 1a displays the ${ }^{1} \mathrm{H}$ NMR spectra of pure PCLT and PPC. The experimental data is consistent with the structure of both polymers and is in correlation with previously reported

Table 2 SEC data of PPC, PCLT and PPC-PCLT co-polymers

\begin{tabular}{llll}
\hline Sample name & $M_{\mathrm{w}}\left(\mathrm{g} \mathrm{mol} \mathrm{mol}^{-1}\right)$ & $M_{\mathrm{n}}\left(\mathrm{g} \mathrm{mol} \mathrm{mo}^{-1}\right)$ & $M_{\mathrm{w}} / M_{\mathrm{n}}$ \\
\hline PPC & 156116 & 44114 & 3.54 \\
PCLT & 2091 & 1620 & 1.29 \\
CP-Seb1 & 99193 & 23671 & 4.19 \\
CP-Seb3 & 65537 & 22588 & 2.90 \\
CP-Suc1 & 92704 & 24143 & 3.84 \\
CP-Suc3 & 111906 & 32198 & 3.47
\end{tabular}


a)
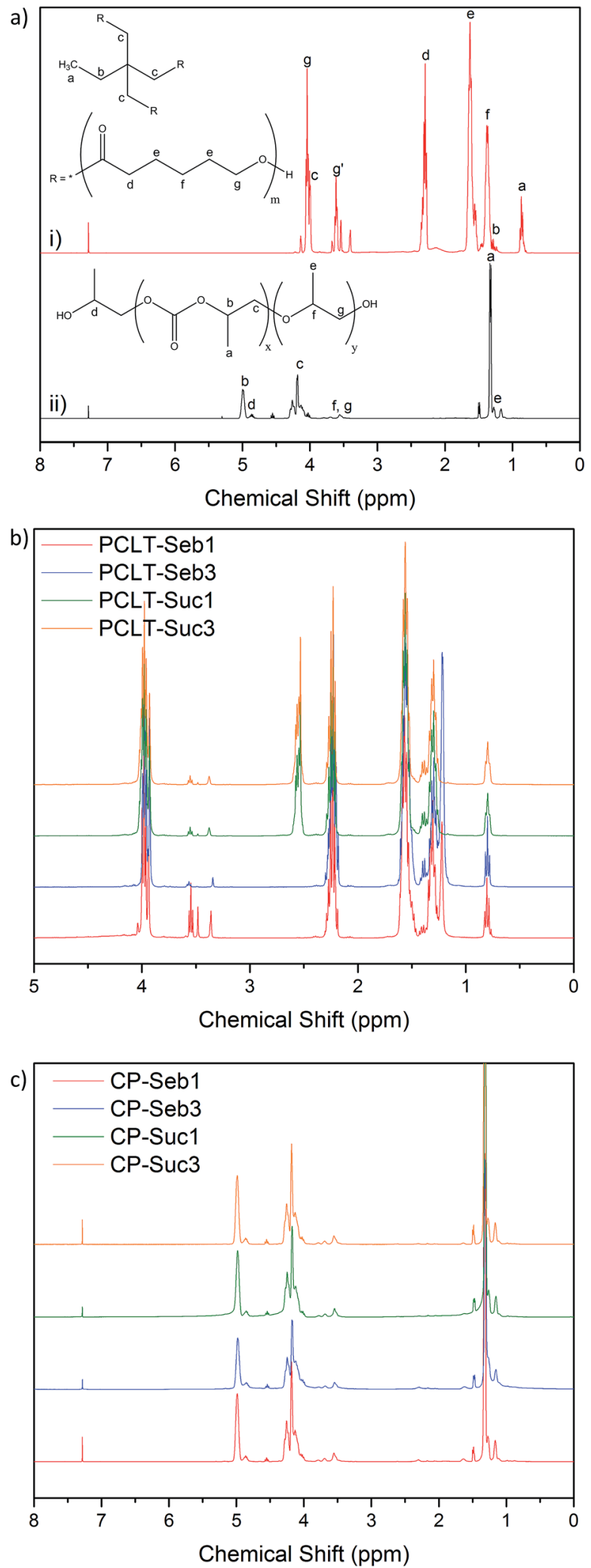

Fig. $1{ }^{1} \mathrm{H}$ NMR spectra; (a) pure polymers (i) PCLT, (ii) PPC, (b) PCLTdiacid pre-polymers, (c) PPC-PCLT co-polymers.

results. ${ }^{25-30}$ The carbonate linkage percentage of PPC was determined to be $98 \mathrm{~mol} \%$, using the peak integration method described by Wang et l. $^{29}$
Peaks a through c correspond to various protons from within the core of PCLT, while peaks $d$ through $g$ are attributed to the various protons within the repeating units. In particular, the peaks $\mathrm{g}^{\prime}$ and $\mathrm{g}$ were assigned to $-\mathrm{CH}_{2}-$ protons next to terminal hydroxyl end groups and esterified $-\mathrm{OH}$ linkages, respectively. ${ }^{31}$ These esterified hydroxyls can be linked to a following repeating unit or, as in this case, covalently bonded with either succinic or sebacic acid. Subsequently, the integrals of peaks $g$ and $g^{\prime}$ were compared to determine the extent of hydroxyl conversion and subsequently confirm the successful reaction between PCLT and succinic/sebacic acid; these results are summarised in Table $\mathrm{S} 1$ in the ESI material, $\uparrow$ while the spectra of the PCLTdiacid pre-polymers are presented in Fig. 1b. Pure PCLT has a $-\mathrm{OH}$ content of $27.0 \%$; this value reduced to 15.2 and $12.0 \%$ following the reaction with 1 mole of sebacic or succinic acid, respectively. As anticipated, the lowest values were obtained following reactions with 3 moles of succinic or sebacic acid (2.4 and $1.9 \%$, respectively). Furthermore, the co-polymer spectra displayed novel peaks corresponding to the specific diacid; PCLT-Seb1 and PCLT-Seb3 exhibited a peak at $1.2 \mathrm{ppm}$, corresponding to the methyl protons of sebacic acid, while PCLTSuc1 and PCLT-Suc3 displayed a peak at $2.5 \mathrm{ppm}$, attributed to the methyl groups within the centre of the succinic acid chain. Likewise, these peak intensities increased with molar ratio. This data confirms successful esterification between the PCLT and diacids, with varying degrees of conversion dependent on the concentration of diacid utilised.

The spectra of the PPC-PCLT co-polymers are displayed in Fig. 1c. The structure of PPC dominates the spectra of all copolymers, due to its greater concentration within the compositions. However, signals corresponding to the PCLT-diacid copolymers can be noticed to a certain degree (e.g. the trace signal at $\sim 2.2 \mathrm{ppm})$. As with the pre-polymers, peak integral of the PPC-PCLT co-polymer spectra were compared to determine the extent of esterification between the PPC and PCLT-diacid pre-polymers. Peaks b (5 ppm) and d (4.9 ppm) from the PPC spectra (refer Fig. 1a) were assigned to $-\mathrm{CH}$ protons adjacent to either the carbonate ester within the repeating unit or terminal hydroxyl groups, respectively. Neither PCLT, succinic acid nor sebacic acid display any overlapping peaks within this region. The co-polymer peak integrals and percentage - $\mathrm{OH}$ are summarised in Table S2 in the ESI material. $\uparrow$ The percentage of $-\mathrm{OH}$ end groups within pure PPC was $11.8 \%$; this dropped to 9.0 and $8.9 \%$ for CP-Seb1 and CP-Suc1, respectively. Thus, the proportion of -OH groups within PPC reduced within the co-polymers, indicating successful covalent bonding with the PCLT-diacid pre-polymer. As anticipated, increasing the degree of substitution within the pre-polymers ultimately provides more sites for esterification within the co-polymers; as a result the lowest - $\mathrm{OH}$ percentages were recorded for CP-Seb3 (8.6\%) and CP-Suc3 (8.0\%). The NMR data confirmed the successful synthesis of both the pre- and co-polymer, while also highlighting the influence of PCLT-diacid degree of substitution on subsequent PPC-PCLT covalent bonding.

ATR-FT-IR. In order to further confirm the successful reactions between (a) PCLT and the dicarboxylic acids and, (b) PCLT-dicarboxylic acid pre-polymer and PPC, ATR-FT-IR was 
utilised. The spectra of pure PCLT and the PCLT pre-polymers are presented in Fig. 2a. Amongst the most prominent peaks characteristic of PCLT's structure include a broad peak at 3445 $\mathrm{cm}^{-1}$ (O-H stretching of hydroxyl groups) and a tall, sharp peak at $1050 \mathrm{~cm}^{-1}$ (C-O stretching of alcohols). ${ }^{32}$ Within all PCLTdicarboxylic acid pre-polymers, both of these peaks significantly diminish or completely disappear. Furthermore, a new, broad band emerged between $\sim 2500$ and $3400 \mathrm{~cm}^{-1}$ within all pre-polymer samples; this was attributed to the $-\mathrm{OH}$ stretching of carboxylic acids. As anticipated, the band was most intense for pre-polymers with a PCLT : dicarboxylic acid ratio of $1: 3$, due to the increased concentration of sebacic- or succinic acid. This behaviour strongly indicates the successful esterification between PCLT and sebacic- or succinic acid, thereby confirming successful pre-polymer formation.

The IR spectra of pure PPC and the PPC-PCLT co-polymers are presented in Fig. 2b. All co-polymers displayed spectra with similar peaks and bands, indicative of both PCLT and PPC
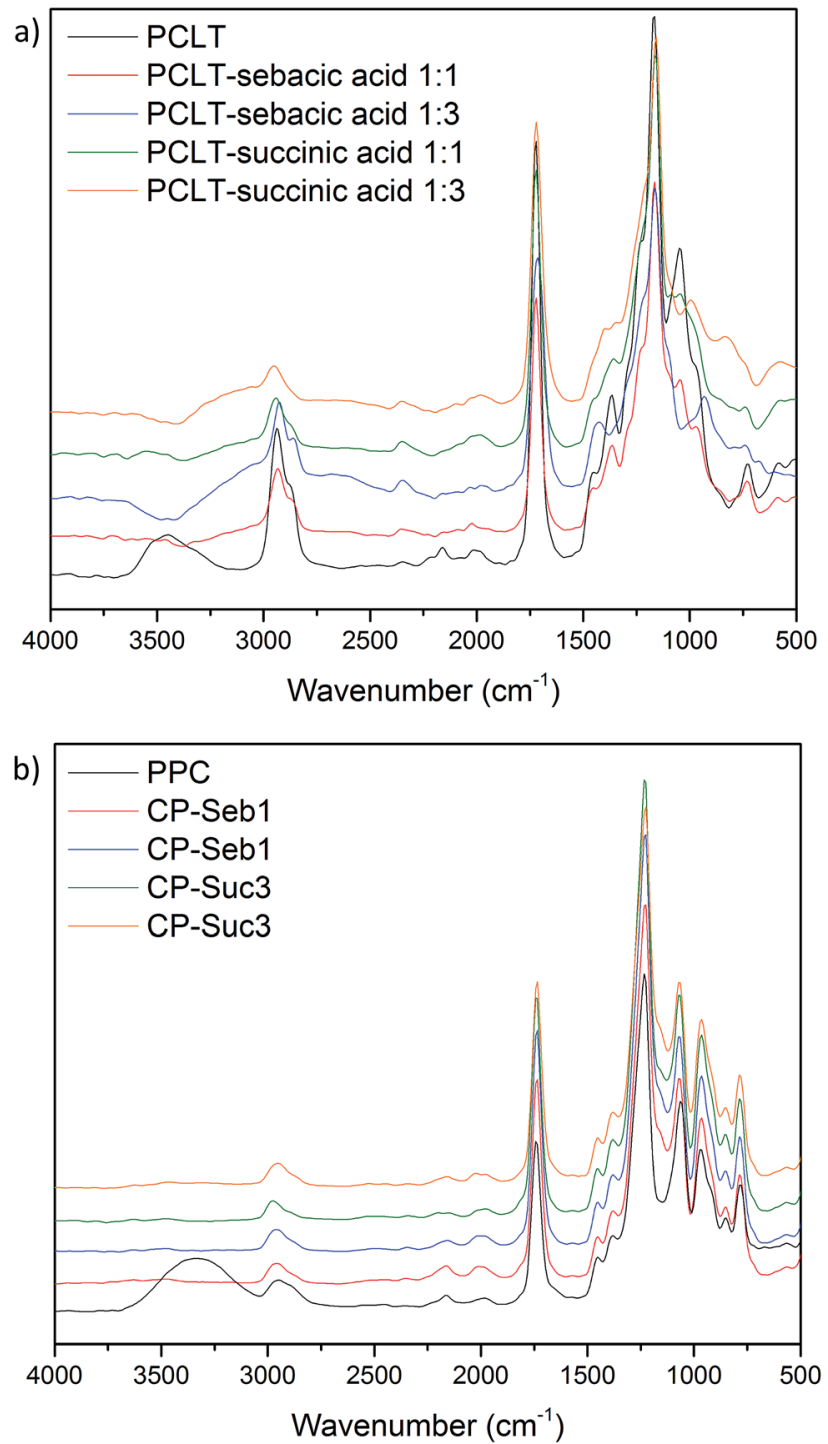

Fig. 2 FT-IR spectra; (a) of pure PCLT and PCLT-dicarboxylic acid prepolymers, (b) pure PPC and PPC-PCLT co-polymers. structure; $2951 \mathrm{~cm}^{-1}$ (C-H stretching), $1736 \mathrm{~cm}^{-1}(\mathrm{C}=\mathrm{O}$ stretching), $1229 \mathrm{~cm}^{-1}$ (O-C-O stretching) and $1070 \mathrm{~cm}^{-1}(\mathrm{C}-\mathrm{O}$ stretching). ${ }^{33,34}$ However, unlike pure PPC, no distinct peak is noticed at $3338 \mathrm{~cm}^{-1}(\mathrm{O}-\mathrm{H}$ stretching and indicative of the presence of hydroxyl end groups on PPC). This behaviour is in correlation with the ${ }^{1} \mathrm{H}$ NMR data and confirms that PCLTdicarboxylic acid pre-polymers and PPC successfully reacted to form PPC-PCLT co-polymers.

\section{Thermal stability and degradation}

The mass loss curves of pure PPC and the PPC-PCLT copolymers are presented in Fig. 3a. The degradation of pure PPC began with a chain unzipping step, attributed to a 'backbiting' attack of the terminal hydroxyl groups on the carbonyl group. ${ }^{35}$ This continues until $\sim 256{ }^{\circ} \mathrm{C}$ where the main mass-loss event corresponding to random chain scission takes place. ${ }^{36}$ All PPC-PCLT displayed a noticeable reduction in mass loss during chain unzipping. As mentioned previously, the unzipping mechanism is dependent on the availability of hydroxyl end groups likely to attack carbonyl groups. Ester linkage formation between the functionalised PCLT and PPC reduces the total
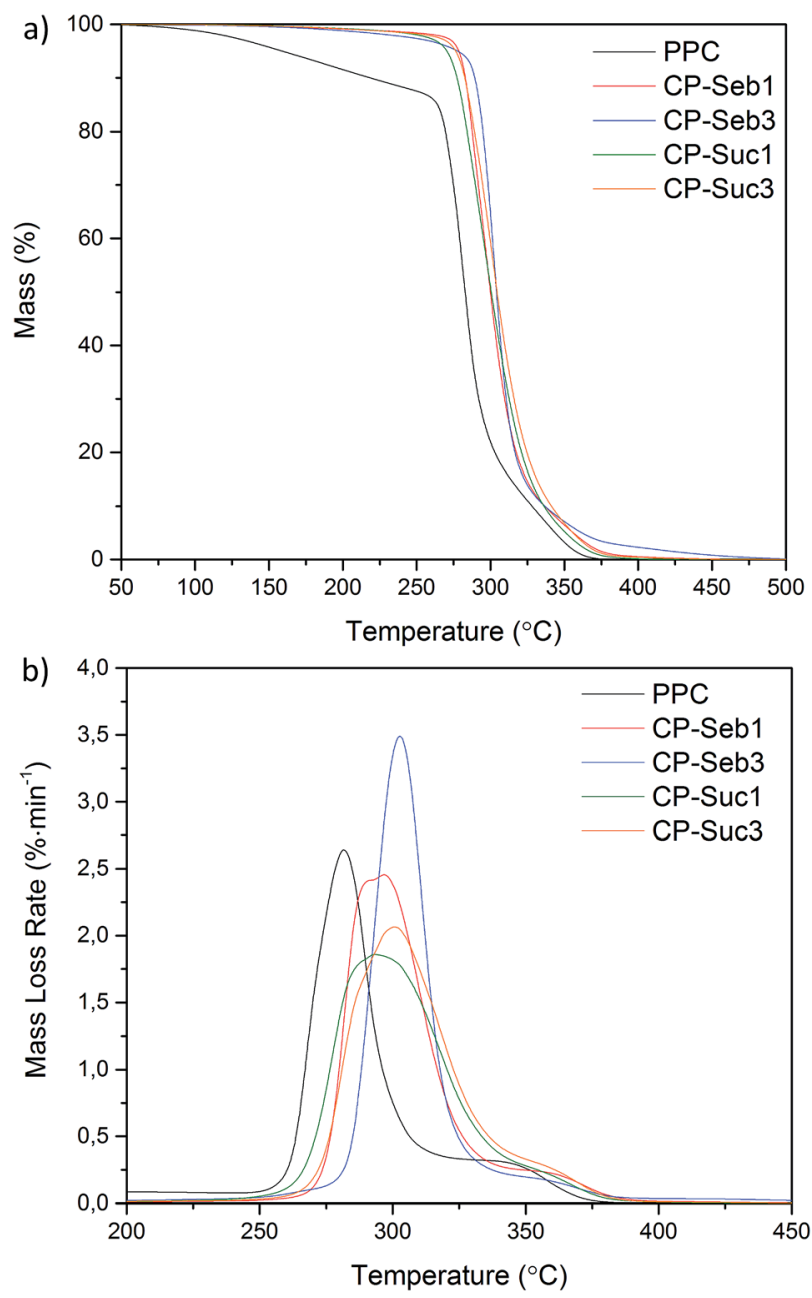

Fig. 3 (a) Mass loss and (b) derivative mass loss curves of pure PPC and PPC-PCLT co-polymers. 
number hydroxyls able to partake in chain unzipping, essentially stabilising the polymer. Furthermore, all PPC-PCLT copolymers underwent chain scission at higher temperatures than pure PPC, as indicated by the curves shifting towards higher temperatures. The introduction of covalent bonds via the terminal hydroxyls of PPC and carboxylic acid end groups of diacid-functionalised PCLT imparts restrictions on the mobility of polymer chain segments. As a result, the amount of energy required for rotation about bonds and subsequent chain scission is increased, leading to increased thermal stability.

In order to further probe the degradation behaviour, as well as characterise the influence of diacid concentration and type, the derivative mass loss curves were taken; these are presented in Fig. 3b. The temperature at which the maximum rate of degradation $\left(T_{\mathrm{d}}\right)$ occurred for PPC was $281{ }^{\circ} \mathrm{C}$; this increased to 293 and $296^{\circ} \mathrm{C}$ for co-polymer CP-Seb1 and CP-Suc1, respectively. Maximum $T_{\mathrm{d}}$ values were observed for CP-Seb3 $\left(303{ }^{\circ} \mathrm{C}\right)$ and CP-Suc3 $\left(300{ }^{\circ} \mathrm{C}\right)$. This corresponds with the aforementioned relationship between increased functionalisation of PCLT leading to increased covalent bonding with PPC and, subsequently, superior thermal stability. When considering the influence of diacid structure, co-polymers containing sebacic acid-functionalised PCLT displayed increased $T_{\mathrm{d}}$ and narrower curves than their succinic acid counterparts. This may be attributed to the boiling point of sebacic acid $\left(294^{\circ} \mathrm{C}\right)$ being higher than that of succinic acid $\left(235^{\circ} \mathrm{C}\right)$, allowing co-polymers containing sebacic acid to display enhanced thermal stability at elevated temperatures. The TGA data highlights the enhanced thermal stability of the PPC-PCLT co-polymers compared with pure PPC, potentially broadening the processability and utilisation of PPC-based polymers, which have been somewhat limited due to poor thermal properties.

\section{Mechanical properties}

Stress-strain analysis. The stress-strain curves of pure PPC and the PCLT-PPC co-polymers are presented in Fig. 4, while the mechanical data is summarised in Table S3 in the ESI. $\dagger$ Pure PPC exhibited a tensile modulus of $0.93 \mathrm{MPa}$, tensile stress of 0.22

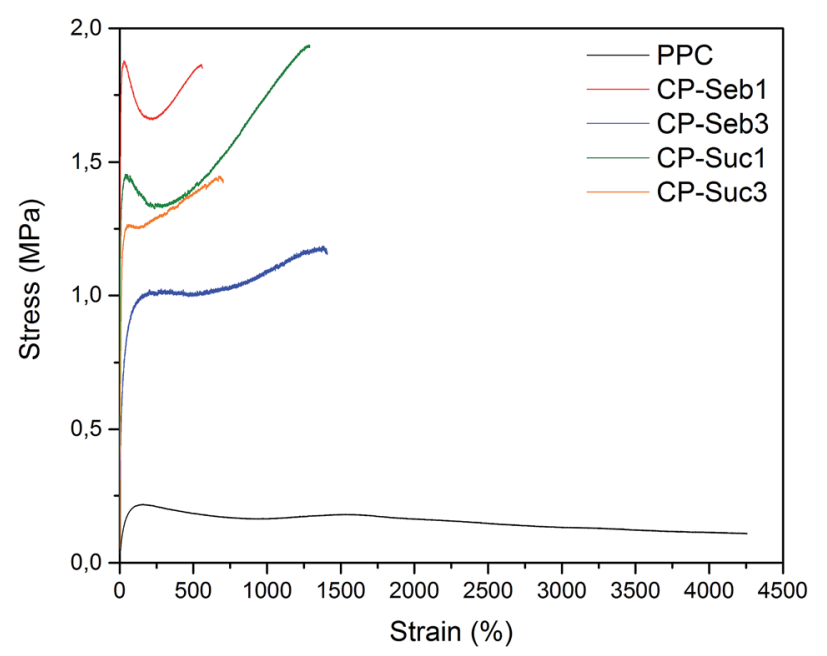

Fig. 4 Stress-strain curves of pure PPC and PPC-PCLT co-polymers.
MPa and elongation of $4260 \%$. It should be noted that the elongation value of PPC is not the elongation at break; rather the experiment was stopped as the Instron tensile tester was approaching its maximum elongation limit. Thus, the extraordinary ductile/elastic properties of PPC (and subsequently its poor strength values and susceptibility to creep) are highlighted. The poor strength values may be attributed to the presence of cyclic propylene carbonate impurities which are a by-product of PPC polymerisation (determined to be $4.0-5.5 \mathrm{~mol} \%$ according to the manufacturer). Since propylene carbonate is a liquid at room temperature, it could impart a plasticising effect on PPC; leading to the low stress and high elongation values.

The stress-strain curves of the PPC-PCLT co-polymers were in significant contrast to that of PPC; a drastic increase in modulus, yield strength and tensile strength were observed within all copolymers. CP-Seb1 displayed the most pronounced enhancements in modulus (58.3 MPa), yield strength $(1.88 \mathrm{MPa})$ and tensile strength (1.85 MPa). These values were $62.7,8.5$ and 8.4 times higher than the respective modulus, yield strength and tensile strength of pure PPC. This is indicative of 'reinforcing' influence of PCLT on PPC; covalent bonding between PCLT and PPC introduces branching into the linear PPC chain, which restrict polymer chain motions and sliding during periods of applied load. Subsequently, strength and rigidity are enhanced at the expense of elongation. Consequently, the elongation at break of the co-polymers was considerably reduced compared to pure PPC. As indicated in the ${ }^{1} \mathrm{H}$ NMR data, a small degree of unreacted sebacic- or succinic acid remained in the co-polymers. Salgado et $a .^{37}$ prepared blended PCL (diol)-sebacic acid gels which were rigid and solid in nature. Thus, residual diacid could potentially contribute towards the increase in strength and stiffness. However, given the relatively high degree of conversion during PCLT functionalisation, the influence of unbound diacid can be considered minor.

When comparing modulus values, the ratio of PCLT: dicarboxylic acid utilised during pre-polymer synthesis had a distinct influence on the tensile behaviour of the co-polymers. The moduli values of CP-Seb1 (58.3 MPa) and CP-Suc1 (55.8 $\mathrm{MPa}$ ) were markedly higher than those of CP-Seb3 (23.8 MPa) and CP-Suc3 (28.3 MPa). Furthermore, the tensile and yield strength of CP-Seb1 and CP-Suc1 were greater than their 3 mole pre-polymer counterparts (CP-Seb3 and CP-Suc3, respectively). Within copolymers containing PCLT reacted with 3 moles of diacid, the degree of covalent linkages with PPC is increased, as confirmed with NMR. This excessive bonding may impart brittleness into the co-polymers. In contrast, co-polymers containing PCLT functionalised with 1 mole of diacid allow reinforcement to be imparted without excessive restrictions on polymer chain segment motions.

All co-polymers experienced strain hardening following yielding. Within amorphous polymers (such as PPC), strain hardening believed to result from segments between entanglements being pulled taut during load application; this leads to an increase in free energy due to reduced configurational entropy. ${ }^{38}$ Through the addition of functionalised PCLT, entanglements are introduced between PPC chain segments. Subsequently, the strain hardening behaviour of the co-polymers is more pronounced than pure PPC. Sub- $T_{\mathrm{g}}$ annealing can also enhance 
the effects of strain hardening, due to reduction in free volume and internal energy changes. ${ }^{39}$ Table S4 in the ESI $\dagger$ displays the glass transition $\left(T_{\mathrm{g}}\right)$ values of pure PPC and the co-polymers obtained via DMA. The $T_{\mathrm{g}}$ of all polymers ranged from $37-46{ }^{\circ} \mathrm{C}$, while the storage and testing temperature was $23{ }^{\circ} \mathrm{C}$. Thus, pure PPC and the co-polymers were effectively annealed, which can contribute to the strain hardening behaviour. It is worth noting that CP-Seb1 and CP-Seb3 had the lowest $T_{\mathrm{g}}$ values, 42 and $41{ }^{\circ} \mathrm{C}$ respectively. Due to the close proximity of the $T_{\mathrm{g}}$ and testing temperature, limited viscous flow may also partly contribute to the high degree of strain hardening exhibited by these two polymers.

As shown in Fig. 4, CP-Seb1 exhibited significantly higher yield strength and tensile strength values than CP-Seb3, while the contrast between CP-Suc1 and CP-Suc3 was less drastic. This difference in magnitude of tensile properties may be attributed to the influence of diacid chain length. The incorporation of succinic acid introduces a 4-carbon chain into the polymer structure, which may have negligible difference on material properties due to the small size. In contrast, sebacic acid contains a significantlylonger 10-carbon chain. This increase in chain length promotes chain entanglement and secondary interactions between polymer segments, leading to enhanced strength. Although CP-Seb3 displayed the lowest tensile- and yield strength values, this was attributed to brittleness derived from excessive covalent bonding. The tensile data indicates that co-polymer modulus (stiffness) and yielding behaviour is primarily influenced by the degree of covalent bonding, while strength is dependent on the length of the diacid used during pre-polymer synthesis.

Creep-recovery. The creep-recovery curves of pure PPC and the PCLT-PPC co-polymers are presented in Fig. 5. As anticipated, pure PPC exhibited the greatest degree of creep, while also the greatest extent of recovery (strain was $-3.2 \%$ at $150 \mathrm{~min}$ ). This behaviour was indicative of PPC's susceptibility to cold flow, stemming primarily from its weak interchain interactions ${ }^{\mathbf{4 0}}$ and $T_{\mathrm{g}}$ being close to room temperature. All PPC-PCLT displayed reduced creep as well as increased permanent deformation; this was attributed to the restrictions of chain segmental motion and sliding, facilitated via covalent bonding between PPC and PCLT pre-polymer. Furthermore, the PPC-PCLT co-polymers were able to be moulded into various shapes which maintained their structure and form. A video demonstrating this phenomena is available in the ESI material. $\dagger$ In contrast, a PPC-PCLT blend prepared with pure, unfunctionalised PCLT did not show the same mouldable characteristics. Although the incorporation of pure PCLT had a toughening influence on PPC, interactions are limited to hydrogen-bonding and entanglements. These weaker interactions are insufficient in restricting flow to the extent of imparting mouldability; covalent linkages are necessary. Thus, with a relatively small concentration of PCLT pre-polymer, cold flow (both with and without applied load) can be drastically retarded.

As the functionalisation of the PCLT pre-polymer increased from 1 mole to 3 moles of succinic or sebacic acid, creep reduced while permanent deformation increased. This was anticipated, as the greater number of carboxylic acid groups imparted onto PCLT increases the number of sites available for potential

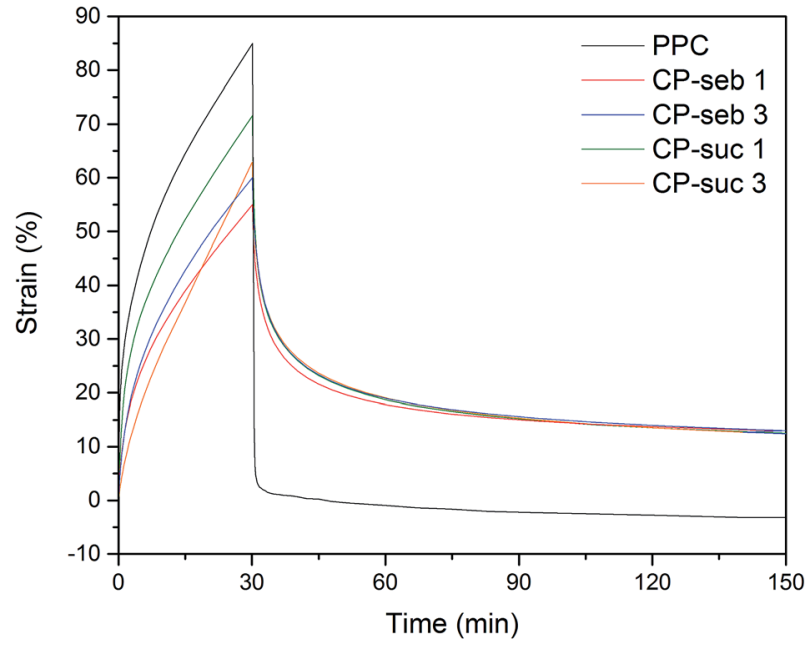

Fig. 5 Creep-recovery curves of PPC and PPC-PCLT co-polymers.

esterification with PPC. Subsequently, a greater degree of covalent bonding leads to more restrictions on chain motion. Conversely, co-polymers functionalised with sebacic acid experienced less creep and more permanent deformation than their succinic acid-functionalised counterparts. One proposed mechanism stems from the difference in diacid chain length on the PCLT pre-polymer; the longer, 10-carbon chain of sebacic acid may encourage chain entanglement and secondary interactions amongst PPC, leading to increased hindrance on segmental motion. In contrast, such aforementioned phenomena may be less likely with shorter, 4-carbon chain succinic acid.

DMA. The storage moduli $\left(E^{\prime}\right)$ of PPC and the co-polymers as a function of temperature are presented in Fig. 6a. Pure PPC exhibited an $E^{\prime}$ value of $905 \mathrm{MPa}$ at $-100{ }^{\circ} \mathrm{C}$. Upon heating the modulus begins to decrease and three transitions were observed; these are more evident within the $\tan (\delta)$ curves, displayed in Fig. $6 \mathrm{~b}$ and c. The four transitions occur at $-75\left(T_{\delta}\right),-38\left(T_{\gamma}\right)$, $-28\left(T_{\beta}\right)$ and $36^{\circ} \mathrm{C}$ (glass transition temperature, $T_{\mathrm{g}}$ ). To the best of the authors' knowledge, there is no reported material concerning the sub- $T_{\mathrm{g}}$ transitions of PPC. However, one proposal is that the $\delta$-, $\gamma$ - and $\beta$-transitions are related to limited motions along the PPC main chain ( $\delta$-transition) and rotation/wagging of the pendant methyl groups ( $\gamma$ - and $\beta$-transitions). ${ }^{\mathbf{4 1}}$

All co-polymers displayed increased $E^{\prime}$ values relative to pure PPC, indicative of increased rigidity and stiffness within the copolymers. Furthermore, PCLT has a $T_{\mathrm{m}}$ of $\sim 6{ }^{\circ} \mathrm{C}$; crystalline domains may contribute towards the storage modulus at upon cooling below $T_{\mathrm{m}}$. For specimens CP-Seb1 and CP-Seb3, increasing the larger $E^{\prime}$ value of the latter is attributed to the greater degree of substitution on the functionalised PCLT precursor. Due to the increased number of reactive sites on PCLT able to covalently bond with PPC, the diacid-functionalised PCLT can impart a greater degree of restrictions/hindrances on PPCchain motion. Furthermore, the increased length of sebacic acid segments may encourage entanglement formation, leading to further chain restrictions and subsequent enhanced stiffness. This can account for CP-Seb3 displaying a higher $E^{\prime}$ than CPSuc3. 

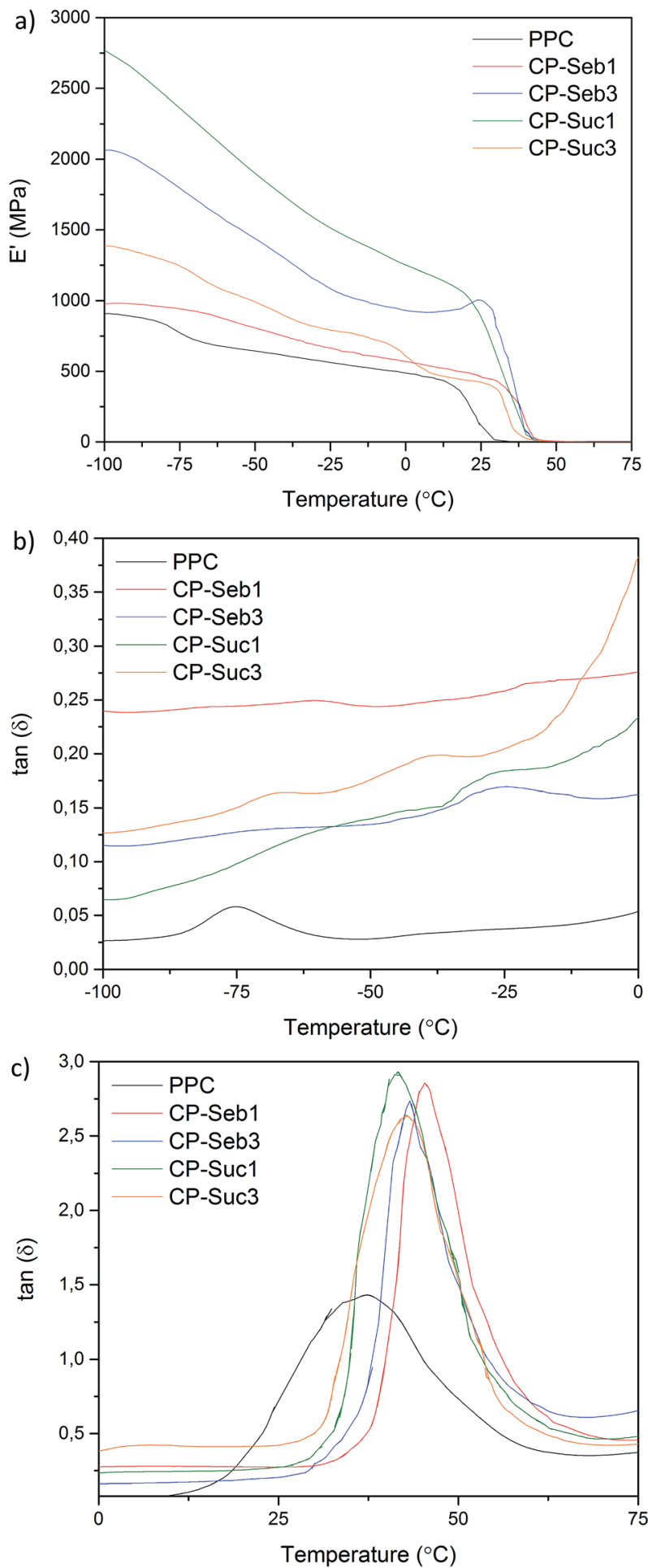

Fig. 6 DMA curves of pure PPC and PPC-PCLT co-polymers; (a) storage moduli, (b) $\tan (\delta)$ within the sub- $T_{\mathrm{g}}$ (PPC) region, (c) $\tan (\delta)$ glass transition region of PPC.

CP-Suc1 displayed the highest $E^{\prime}$ values, while CP-Suc3 displayed a reduction in storage modulus; the behaviour being opposite to that displayed by co-polymers CP-Seb1 and CP-Seb3. It is worth noting that the tensile moduli of CP-Suc1 obtained during stress-strain analysis was similar to that of CP-Seb1 (refer to Table S3 in the ESI material $\dagger$ ). This behaviour may be attributed to the shorter succinic acid chains imparting a brittling influence at higher degrees of substitution (as is the case with CP-Suc3). However, at lower degrees of substitution (as with CP-Suc1), successful reinforcement may be attainable without excessive restriction on PPC chain segment motion.

The maximum from the $\tan (\delta)$ curves was used to indicate the $T_{g}$; these values and other thermal transitions are summarised in Table S4 in the ESI. $\uparrow$ Pure PPC displayed a $T_{\mathrm{g}}$ of 37 ${ }^{\circ} \mathrm{C}$; this increased for all co-polymers, with CP-Seb1 yielding the highest $T_{\mathrm{g}}$ of $46{ }^{\circ} \mathrm{C}$. At temperatures below the $T_{\mathrm{g}}$ peak, copolymer spectra displayed increased $\tan (\delta)$ heights relative to pure PPC. These observations are indicative of increased restrictions on segmental motion of PPC; via covalent bonding and physical entanglements. ${ }^{42}$ Co-polymers prepared with sebacic acid displayed higher $T_{\mathrm{g}}$ values than their succinic acid counterparts. This is attributed to the increased chain length of sebacic acid; the probability of chain entanglement formation increases with chain length, resulting in a greater degree of restrictions being imparted on PPC chain motion.

Co-polymers showed a significant increase in $\tan (\delta)$ peak intensity, indicative of enhanced damping properties. The damping ability of a polymer is believed to be influenced by two factors; (a) the amount of free volume, (b) internal friction between macromolecules across the glass transition region. ${ }^{43}$ Considering these two criteria, two possible explanations for the enhanced damping properties are proposed. Firstly, covalent bonding between the three-armed functionalised PCLT and linear PPC introduces a three-dimensional network structure to the system. This may be compared with branching ${ }^{44}$ or crosslinking ${ }^{45}$ which can entrap free volume within the system (while also enhancing stiffness, as observed in the $E^{\prime}$ curves). Secondly, there may be weak interfacial interaction between entangled segments of PCLT and PPC. This poor interaction is especially vulnerable when subjected to external stresses or if both polymers are in the glassy state ${ }^{46}$ (PPC is amorphous, while the $T_{\mathrm{g}}$ of PPC is greater than the $T_{\mathrm{m}}$ of PCLT). Subsequently, PPC chain motions at the interface may slip more easily, leading to increased free volume and damping ability. ${ }^{47}$

\section{Conclusions}

Co-polymers of PCLT functionalised with either succinic- or sebacic acid and PPC were functionalised utilising a one-pot, two step method. ${ }^{1} \mathrm{H}$ NMR and ATR-FT-IR confirmed the structure and successful synthesis of the co-polymers. Copolymers displayed enhanced thermal stability compared with pure PPC. This was attributed to the esterification reaction between PPC and diacid-functionalised PCLT (a) reducing the number of vacant hydroxyl groups able to participate in chain unzipping, and (b) restricting segmental motion of PPC chain segments. This dramatically broadens the processability and subsequent utilisation potential of PPC. All co-polymers exhibited superior tensile moduli, strength, storage moduli, glass transition temperatures and damping ability than pure PPC, while also displaying reduced creep and flow properties. The ability of the functionalised PCLT to influence PPC 
rheological and cold-flow properties was highlighted by the PPC-PCLT co-polymers being readily mouldable and maintaining a greater degree of dimensional stability compared with pure PPC.

Two factors primarily governed the mechanical properties. Firstly, increasing the degree of substitution of the functionalised PCLT increased the likelihood of ester-linkage formation with PPC. This resulted in greater restrictions on PPC chain motions, reflected in increased rigidity and yielding behaviour. Secondly, functionalising PCLT with the longer sebacic acid increases the likelihood of entanglement formation, leading to further restrictions on PPC chain motion, resulting in enhanced strength properties. These restrictions on chain motion (in the form of ester bonds and entanglements) were evident when load was applied and removed, as demonstrated by the reduced creep behaviour and dimensional stability following handmoulding, respectively. The enhanced damping properties were attributed to pockets of free volume trapped within the copolymer, and reduced internal friction between PCLT and PPC.

The drastic enhancement in thermal stability, tensile behaviour, creep/flow properties and damping ability at relatively low PCLT concentrations provides great potential for further expanding the potential applications of PPC and its blends/co-polymers. Namely, consumer products, packaging, construction and biomedical applications are amongst the most promising application fields worth pursuing.

\section{Notes and references}

1 C. K. Williams and M. A. Hillmyer, Polym. Rev., 2008, 48, 110.

2 L. Montero de Espinosa and M. A. R. Meier, Eur. Polym. J., 2011, 47, 837-852.

3 G. Kim, M. Ree, H. Kim, I. Kim, J. Kim and J. Lee, Macromol. Res., 2008, 16, 473-480.

4 X. C. Ge, X. H. Li, Q. Zhu, L. Li and Y. Z. Meng, Polym. Eng. Sci., 2004, 44, 2134-2140.

5 X. C. Ge, Y. Xu, Y. Z. Meng and R. K. Y. Li, Compos. Sci. Technol., 2005, 65, 2219-2225.

6 X. Jing, M. R. Salick, T. Cordie, H. Mi, X. Peng and L. Turng, Ind. Eng. Chem. Res., 2014, 53, 9391-9400.

7 G. Flodberg, I. Helland, L. Thomsson and S. Bodil Fredriksen, Eur. Polym. J., 2015, 63, 217-226.

8 L. Huihong, P. Lisha, L. Qiang, X. Nai, L. Lingbin, P. Sujuan and F. Songbao, e-Polym., 2010, 038, 1-9.

9 X. Wu, H. Zhao, B. Nörnberg, P. Theato and G. A. Luinstra, Macromolecules, 2014, 47, 492-497.

10 G. Luinstra and E. Borchardt, Adv. Polym. Sci., 2012, 245, 2948.

11 D. V. Mistura, A. D. Messias, E. A. R. Duek and M. A. T. Duarte, Artif. Organs, 2013, 37, 978-984.

12 M. Duarte, A. Motta and E. Duek, Int. J. Polym. Sci., 2014, 2014, 1-9.

13 Z. Li, Z. Zhang, K. L. Liu, X. Ni and J. Li, Biomacromolecules, 2012, 13, 3977-3989.

14 T. Wang, F. Huang and S. Lee, Polym. Int., 2002, 51, 13481352.
15 K. Lee, D. Kim and B. Kim, Biotechnol. Bioprocess Eng., 2007, 12, 152-156.

16 N. Castro, P. Goldstein and M. Cooke, Adv. Biosci. Biotechnol., 2011, 2, 167-173.

17 M. Labet and W. Thielemans, Chem. Soc. Rev., 2009, 38, 3484-3504.

18 H. Hatakeyama, Y. Izuta, K. Kobashigawa, S. Hirose and T. Hatakeyama, Macromol. Symp., 1998, 130, 127-138.

19 T. Buntara, S. Noel, P. H. Phua, I. Melián-Cabrera, J. G. deVries and H. J. Heeres, Angew. Chem., Int. Ed., 2011, 50, 7083-7087.

20 Y. Hwang, M. Ree and H. Kim, Catal. Today, 2006, 115, 288294.

21 G. Guo, Y. Wang, J. Deng, R. Fan, L. Zhou, A. Tong, Y. Zheng, X. Jianguo and X. Zhang, RSC Adv., 2015, 5, 82834-82844.

22 S. Chen, B. Chen, J. Fan and J. Feng, ACS Sustainable Chem. Eng., 2015, 3, 2077-2083.

23 P. Song, Y. Shang, S. Chong, X. Zhu, H. Xu and Y. Xiong, RSC Adv. , 2015, 5, 32092-32095.

24 M. Yao, H. Deng, F. Mai, K. Wang, Q. Zhang, F. Chen and Q. Fu, eXPRESS Polym. Lett., 2011, 5, 937-949.

25 H. S. Shirazi, P. K. Forooshani, B. Pingguan-Murphy and I. Djordjevic, Prog. Org. Coat., 2014, 77, 821-829.

26 G. Shi, D. G. Cooper and M. Maric, Polym. Degrad. Stab., 2011, 96, 1639-1647.

27 J. Hilf, P. Schulze, J. Seiwert and H. Frey, Macromol. Rapid Commun., 2014, 35, 198-203.

28 A. Yoshida, S. Honda, H. Goto and H. Sugimoto, Polym. Chem., 2014, 5, 1883-1890.

29 S. Wang, Y. Huang, B. Liao, G. Lin, G. Cong and L. Chen, Int. J. Polym. Anal. Charact., 1997, 3, 131-143.

30 W. Zhang, L. Lu, Y. Cheng, N. Xu, L. Pan, Q. Lin and Y. Wang, Green Chem., 2011, 13, 2701-2703.

31 L. Xue, S. Dai and Z. Li, Macromolecules, 2009, 42, 964-972.

32 K. Muhammad, W. Abas, K. KimI, B. Pingguan-Murphy, N. Zain and H. Akram, Clinics, 2012, 67, 629-638.

33 B. Fei, C. Chen, S. Peng, X. Zhao, X. Wang and L. Dong, Polym. Int., 2004, 53, 2092-2098.

34 L. A. Kanis, E. L. Marques, K. M. Zepon, J. R. Pereira, S. Pamato, M. T. de Oliveira, L. G. Danielski and F. C. Petronilho, J. Biomater. Appl., 2014, 29, 654-661.

35 J. An, Y. Ke, X. Cao, Y. Ma and F. Wang, Polym. Chem., 2014, $5,4245-4250$.

36 T. J. Spencer and P. A. Kohl, Polym. Degrad. Stab., 2011, 96, 686-702.

37 C. L. Salgado, E. M. S. Sanchez, C. A. C. Zavaglia and P. L. Granja, J. Biomed. Mater. Res., Part A, 2012, 100, 243251.

38 V. S. Jatin and S. Basu, Int. J. Plast., 2014, 56, 139-155.

39 R. N. Haward, Macromolecules, 1993, 26, 5860-5869.

40 Y. Tao, X. Wang, X. Zhao, J. Li and F. Wang, J. Polym. Sci., Part A: Polym. Chem., 2006, 44, 5329-5336.

41 J. Fried, Polymer Science and Technology, Prentice Hall PTR, 2nd edn, Upper Saddle River, 2003.

42 S. Han and L. Drzal, Curing Characteristics and Water Absorption behaviour of Glucose Based Polymers, in 
Frontiers in Polymer Research, ed. R. Bregg, Nova Sciences Publishers Inc, New York, 2006, pp. 1-26.

43 T. Trakulsujaritchok and D. J. Hourston, Eur. Polym. J., 2006, 42, 2968-2976.

44 D. Ladin, C. Park, S. Park and H. Naguib, SPE ANTEC Technical Papers. 46, 2000, vol. 2, pp. 1955-1977.
45 C. S. Pfeifer, Z. R. Shelton, R. R. Braga, D. Windmoller, J. C. Machado and J. W. Stansbury, Eur. Polym. J., 2011, 47, 162-170.

46 D. P. Varma, Ph.D thesis, Cochin University of Science and Technology, 2010, p. 127.

47 R. Ramani and S. Alam, J. Appl. Polym. Sci., 2016, 133, 4296142969. 